\title{
La satisfacción y desempeño laboral en los trabajadores de la Empresa LSA ENTERPRISES PERU SAC, en la localidad de Carquín
}

\author{
Satisfaction and Work Performance in the workers of the Company LSA ENTERPRISES PERU \\ $\mathrm{SAC}$, in the town of Carquin \\ Wilmer Huerta Hidalgo', Gleny Amelia Ching Campos²
}

\section{RESUMEN}

Objetivo: El presente trabajo de investigación tuvo como objetivo determinar la relación entre la satisfacción laboral y el desempeño laboral en los trabajadores de la empresa LSAENTERPRISES PERU SAC, en la localidad de Carquin 2017. Material y Métodos: Por ser un estudio de nivel descriptivo y correlacional, de tipo aplicada, la cual tiene como hipótesis que existe relación entre la satisfacción laboral y el desempeño laboral en los trabajadores de la empresa LSA ENTERPRISES PERU SAC, localidad de Carquin 2017, Se utilizó la técnica de la encuesta, teniendo como instrumento el cuestionario. El procesamiento estadístico de los datos se realizó por medio de la aplicación del paquete estadístico SPSS en su versión 21. Se trabajó con una muestra de 80 trabajadores. Resultados: En esta investigación se encontró que existe relación entre la satisfacción y desempeño laboral en los trabajadores de la empresa LSA ENTERPRISES PERU SAC, localidad de Carquin 2017. Conclusiones: Puedo mencionar que cuando los trabajadores se siente con más satisfacción, motivados su desempeño laboral aumenta, también el rendimiento de los trabajadores aumenta, quiere decir que un trabajador satisfecho y bien motivado tendrá un mejor rendimiento en su trabajo.

Palabras clave: Rendimiento, Satisfacción, Desempeño, Laboral, Trabajadores.

\section{ABSTRACT}

Objective: The objective of this research was to determine the relationship between job satisfaction and job performance in the workers of the company LSA ENTERPRISES PERU SAC, in the town of Carquin 2017. Material and Methods: $s$ it is a descriptive and correlational level study, of applied type, which has as hypothesis that there is a relationship between job satisfaction and work performance in the workers of the company LSA ENTERPRISES PERU SAC, locality of Carquin 2017, The survey technique was used, having as an instrument the questionnaire. The statistical processing of the data was carried out through the application of the statistical package SPSS in its version 21. Results: We worked with a sample of 80 workers, in this research it was found that there is a relationship between satisfaction and work performance in the workers of the company LSA ENTERPRISES PERU SAC, locality of Carquin 2017. Conclusions: I can mention that when workers feel more satisfied, motivated their work performance increases, also the performance of workers increases, means that a satisfied and well motivated worker will have a better performance in your work.

Keywords: Performance, Satisfaction, Performance, Labor, Workers.

\section{INTRODUCCIÓN}

En esta última década, en el mundo de las organizaciones, para poder cumplir con ciertos objetivos o metas, es necesaria cambiar la noción sobre el ser humano, y en la actualidad, ya no se mantiene la idea que el obrero o empleado responde de modo automático a las exigencias de las organizaciones, por el contrario, se asume que los trabajadores son recursos humanos de alta relevancia, que son más bien, sustento del funcionamiento y desarrollo de los sistemas organizacionales.

También se observa que las empresas ofrecen sus productos a los clientes pero no se preocupan por los problemas que agobian a sus empleados en el entorno de la empresa, ya que ellos necesitan desarrollar sus habilidades, y de esta manera poder sentirse satisfechos con lo que realizan dentro de la empresa, visto que existe un gran aceptación al respecto a que la satisfacción laboral es una variable fundamental dentro de la productividad de la empresa.

El activo más importante de la organización son los empleados (colaboradores) y es necesario generar satisfacción en los mismo tanto de carácter económico como en las condiciones de trabajo, lo cual tienen implicancia en la productividad y rentabilidad de la

$$
\text { Recibido 29/08/2021 Aprobado 26/09/2021 }
$$

Este es un artículo de acceso abierto, distribuido bajo los términos de la Licencia Creative Commons Atribución 4.0 Internacional (http://creativecommons.org/licenses/by/4.0/)

\section{(c) (i)}

${ }^{1} \mathrm{M}(\mathrm{o})$ en Administración estratégica, Licenciado en administración, Docente Universidad Nacional José Faustino Sánchez Carrión, Huacho, Huaura, Lima, Perú ORCID: 0000-0002-8772-8672. Email: whuertah@unjfsc.edu.pe

${ }^{2} \mathrm{M}(\mathrm{o})$ en Administración estratégica, Licenciada en administración, Docente Universidad Nacional José Faustino Sánchez Carrión, Huacho, Huaura, Lima, Perú. ORCID: 0000-0001-7152-9110. Email: gching@unjfsc.edu.pe 


\section{INTRODUCCIÓN}

En esta última década, en el mundo de las organizaciones, para poder cumplir con ciertos objetivos o metas, es necesaria cambiar la noción sobre el ser humano, y en la actualidad, ya no se mantiene la idea que el obrero o empleado responde de modo automático a las exigencias de las organizaciones, por el contrario, se asume que los trabajadores son recursos humanos de alta relevancia, que son más bien, sustento del funcionamiento y desarrollo de los sistemas organizacionales.

También se observa que las empresas ofrecen sus productos a los clientes pero no se preocupan por los problemas que agobian a sus empleados en el entorno de la empresa, ya que ellos necesitan desarrollar sus habilidades, y de esta manera poder sentirse satisfechos con lo que realizan dentro de la empresa, visto que existe un gran aceptación al respecto a que la satisfacción laboral es una variable fundamental dentro de la productividad de la empresa.

El activo más importante de la organización son los empleados (colaboradores) y es necesario generar satisfacción en los mismo tanto de carácter económico como en las condiciones de trabajo, lo cual tienen implicancia en la productividad y rentabilidad de la empresa (Talento Humano).

El Sector Pesquero en la localidad de CARQUÍN, está conformado por empresas importantes tales como: EXALMAR y LSA ENTERPRISES PERU SAC, cada una de ellas busca reducir costos y ser más productivos para logara la competitividad dentro del mercado.

La Empresa LSA ENTERPRISES PERU SAC, tiene su oficina principal en Lima - San Isidro, y la Pesquera que tiene en la localidad de CARQUíN, la cual está dedicada a la producción de harina y aceite de pescado.

Este trabajo está relacionado al nivel de satisfacción y desempeño laboral que tiene sus trabajadores de la empresa LSA ENTERPRISES PERU SAC, la cual evaluaran cada una de las áreas que tenga la empresa y se propondrán alternativas para poder mejorar la satisfacción y el desempeño laboral.

\section{MATERIAL Y MÉTODOS}

Tipo de investigación: Aplicada

Nivel de investigación: descriptivo - correlacional

Diseño de investigación: transversal - descriptivo correlacional.

La población está conformada por 80 trabajadores de la empresa LSA ENTERPRISES PERU SAC, localidad de Carquín. y La muestra es finita por lo cual se tomó toda la población.

Técnicas de recolección
Encuesta: con esta técnica vamos poder obtener información generalmente de una muestra de sujetos, mediante preguntas realizadas de la misma manera, con el fin de obtener información acerca de nuestro proyecto de investigación.

Instrumento: fue la Escala de Satisfacción laboral elaborado por Sonia Palma, SL-SPC (1999). El cual es una escala tipo Likert modificada, el cual tiene una confiabilidad y validez, consta de 36 items.

Técnicas para el procesamiento de la información

Básicamente el estudio comprenderá tres etapas:

Primera, destinada a la recolección de información general, revisión rápida de estudios realizados, textos, publicaciones oficiales, informes estadísticos, búsquedas por Internet de publicaciones electrónicas, visita a bibliotecas de instituciones relacionadas con el tema. Esta etapa se concluirá con la aprobación del proyecto de tesis por la universidad.

Segunda, consistirá en la preparación de materiales para la recopilación de datos en campo. Entre éstas el diseño, elaboración del instrumento y aplicación de los cuestionarios a una muestra representativa de la población objeto de estudio.

Tercera, consistirá en el procesamiento e interpretación de los datos recopilados.

\section{RESULTADOS}

Tabla 1

Correlación entre Satisfacción Laboral y Desempeño Laboral

\begin{tabular}{llrr}
\hline & & $\begin{array}{c}\text { Satisfacción } \\
\text { Laboral }\end{array}$ & $\begin{array}{c}\text { Desempeño } \\
\text { Laboral }\end{array}$ \\
\hline \multirow{2}{*}{$\begin{array}{l}\text { Satisfacción } \\
\text { Laboral }\end{array}$} & Pearson & 1 & $0,221^{*}$ \\
& Sig. (bilateral) & & 0,049 \\
& $\mathrm{~N}$ & 80 & 80 \\
& Correlación de & $0,221^{*}$ & 1 \\
Desempeño & Pearson & & \\
Laboral & Sig. (bilateral) & 0,049 & \\
& $\mathrm{~N}$ & 80 & \\
\hline
\end{tabular}

*. La correlación es significante al nivel 0,05 (bilateral).

En la tabla 1, se puede observar que existe una correlación significante entre la satisfacción laboral y el desempeño laboral, la cual tiene sig, de ,049. Se puede concluir que ha mayor satisfacción, el desempeño laboral aumentara esta se puede ver en la correlación que encontramos, que cuando un trabajador está satisfecho en su trabajo, el desempeño aumentara ya que trabajara con un mayor rendimiento y según nuestra investigación los trabajadores de la empresa LSAENTERPRISES 
, se encuentra parcialmente satisfecho con su trabajo.

\section{Tabla 2}

Correlación entre Satisfacción Laboral y Producción Laboral

\begin{tabular}{llcr}
\hline & & $\begin{array}{c}\text { Satisfacción } \\
\text { Laboral }\end{array}$ & $\begin{array}{r}\text { Productividad } \\
\text { Laboral }\end{array}$ \\
\hline & Correlación & 1 & $0,270^{*}$ \\
Satisfacción & de Pearson & & \\
Laboral & Sig. (bilateral) & & 0,016 \\
& $\mathrm{~N}$ & 80 & 1 \\
& Correlación & $0,270^{*}$ & 80 \\
Productividad & de Pearson & & \\
Laboral & Sig. (bilateral) & 0,016 & \\
& $\mathrm{~N}$ & 80 &
\end{tabular}

*. La correlación es significante al nivel 0,05 (bilateral).

En la tabla 2, se puede observar que existe una correlación significante entre la satisfacción laboral y la Productividad laboral, la cual tiene sig, de ,016. Los trabajadores de la empresa LSA ENTERPRISES PERU SAC mayormente salen insatisfechos por la falta de incentivos, motivacionales (no pagan a tiempo, aumento de horas de trabajo, falta de respeto de los superiores hacia los subordinados), y estos factores determinan la calidad de trabajo o Producción Laboral por parte de los trabajadores.

\section{Tabla 3}

Correlación entre Satisfacción Laboral y Eficacia

\begin{tabular}{clcc}
\hline & & $\begin{array}{c}\text { Satisfacción } \\
\text { Laboral }\end{array}$ & Eficacia \\
\hline $\begin{array}{c}\text { Satisfacción } \\
\text { Laboral }\end{array}$ & $\begin{array}{l}\text { Pearson } \\
\text { Sig. (bilateral) }\end{array}$ & 1 & $0,235^{*}$ \\
& $\mathrm{~N}$ & 80 & 0,036 \\
& Correlación de & $0,235^{*}$ & 1 \\
Eficacia & Pearson & & \\
& Sig. (bilateral) & 0,036 & \\
& $\mathrm{~N}$ & 80 & 80 \\
\hline
\end{tabular}

*. La correlación es significante al nivel 0,05 (bilateral).

En la tabla 54, se puede observar que existe una correlación significante entre la satisfacción laboral y la eficacia, la cual tiene sig, de , 018 .
Tabla 4

Correlación entre satisfacción laboral y eficiencia laboral

\begin{tabular}{llrr}
\hline & & $\begin{array}{c}\text { Satisfacción } \\
\text { Laboral }\end{array}$ & \multicolumn{2}{c}{$\begin{array}{c}\text { Eficiencia } \\
\text { Laboral }\end{array}$} \\
\hline \multirow{2}{*}{$\begin{array}{l}\text { Satisfacción } \\
\text { Laboral }\end{array}$} & Correlación de & 1 & 0,055 \\
& Pearson & & 0,629 \\
& Sig. (bilateral) & & 80 \\
& $\mathrm{~N}$ & 80 & 1 \\
& Correlación de &,- 055 & \\
Eficiencia & Pearson & & \\
Laboral & Sig. (bilateral) &, 629 & 80 \\
& $\mathrm{~N}$ & 80 & \\
\hline
\end{tabular}

En la tabla 4, se puede observar que no existe una correlación entre la satisfacción laboral y la Eficiencia laboral.

El propósitos de esta investigación es para mejora la calidad de vida de los colaboradores de la empresa LSA ENTERPRISES PERU SAC, ya que tener al personal insatisfecho no ayuda a la organización en ningún sentido, por eso es bueno tener en cuenta cómo se siente los colaboradores dentro de su centro de trabajo.

\section{DISCUSIONES}

A partir de los hallazgos encontrados en la tesis "Clima Organizacional y Satisfacción Laboral en la Asociación para el desarrollo empresarial en Apurímac, Andahuaylas, 2015", realizado por Quispe Aquino, 2015 en la Universidad Nacional José María Arguedas, La hipótesis principal señalaba que existía relación entre el clima organizacional y satisfacción laboral. La principal conclusión comprobó que hay relación entre las dos variable, es decir, existe relación significativa positiva entre el clima organizacional y satisfacción laboral. A nivel de las hipótesis específicas se comprobó las dimensiones de clima organizacional la estructura, autonomía, relaciones interpersonales, identidad se correlacionaron de forma significante y positiva con la satisfacción laboral. Sin embargo no se encontró relación entre la dimensión recompensa con la satisfacción laboral en la Asociación para el Desarrollo Empresarial el Apurímac.

En la tesis "Clima, Motivación Intrínseca y Satisfacción Laboral en Trabajadores de Diferentes Niveles Jerárquicos. Lima, Junio 2014"; realizado por Carlos Dávila 2014, en la Pontífice Universidad Católica del Perú; Los resultados muestran una correlación significativa y positiva entre las variables clima organizacional, motivación intrínseca y satisfacción laboral (100) entre .40 y .58, p<.01). Existen también, en los diferentes grupos jerárquicos, diferencias estadísticamente significativas entre las variables 
estudiadas. Los trabajadores que ocupan posiciones más elevadas dentro de la organización, perciben el clima organizacional de manera más favorable, reportan niveles más altos de motivación intrínseca y satisfacción laboral (4.00, 5.74 y 4.47 respectivamente).

En la tesis "Clima Organizacional y Satisfacción Laboral en enfermeros de unidades criticas del Hospital Nacional Dos de Mayo 2012", realizado porCalcina Cáceres 2014, en la Universidad Nacional Mayor de San Marcos; los resultados. Del $100 \%$ (35), en el Clima Organizacional $71.4 \%$ (25) fue regular y $28.6 \%$ (10) muy bueno. Respecto a la dimensión responsabilidad $60 \%$ (21), recompensa 83\% (29), apoyo 83\% (29), estándares de desempeño $51 \%$ (18), y conflicto $83 \%$ (29) manifestaron que fue regular; seguido de un $57 \%$ (20) que expresa que es muy bueno la estructura, $57 \%(20)$ el riesgo, $37 \%$ (13) la dimensión calor y $71 \%$ (25) en la dimensión identidad. Según la satisfacción laboral 85.7\% (30) fue media, 8.6\% (3) alta, y 5.7\% (2) baja. Respecto al factor condiciones físicas y materiales $68.6 \%$ (24), beneficios laborales $57 \%$ (20), políticas administrativas $91.4 \%$ (32), relaciones sociales $91.4 \%$ (32), desarrollo personal $68.6 \%$ (24), desempeño de las tareas $77.1 \%$ (27), relación con la autoridad $62.9 \%$ (22), el mayor porcentaje y mayoría expresan que la satisfacción fue media. Conclusiones.

El clima organizacional según los enfermeros de Unidades Críticas en su mayoría son regular referida a la recompensa, apoyo y conflicto, y un porcentaje considerable expresa que es muy buena en cuanto a estructura, riesgo y dimensión calor. En cuanto a la satisfacción laboral el mayor porcentaje y la mayoría manifiestan que es media referida a las condiciones físicas y materiales, beneficios sociales, relaciones sociales, desarrollo personal y desempeño de las tareas.

Después de haber revisado sus resultados de las investigaciones anteriores podemos deducir que a mayor satisfacción mejor desempeño laboral, la cual se busca que los trabajadores este motivados para que realicen sus trabajo con eficiencia y eficacia, en esta investigación quiero aportar en el mejoramiento del desempeño laboral en los trabajadores de la empresa LSA ENTERPRISES PERU SAC, la cual buscara mejorar sus plan de capacitación y el de remuneraciones para que sus trabajadores estén satisfechos de ello.

\section{AGRADECIMIENTO}

Agradezco a Dios, a mis padres y a todos los que me apoyaron para poder terminar este proyecto.

\section{REFERENCIAS BIBLIOGRÁFICAS}

Apuy Arias, L. I. (2008). Factores del clima organizacional que influyen en la satisfaccion laboral del personal de enfermeria, en el servicio de emergencias del Hospital San Rafael de Alajuela, Junio - Noviembre 2008. San Jose, Costa Rica.

Arce Ortiz, Elmer (2013). Derecho individual del trabajo en el Perú(1 ra edición). Perú: Editorial Palestra.

Arévalo Vela, Javier (2004). Manual de Legislación Laboral Tomo II (1ra edición). Perú, Editorial Cultural Cuzco.

Arredondo Baquerizo, Digna, "Inteligencia emocional y clima organizacional en el personal del Hospital "Félix Mayorca Soto". En Perú, 2008.

Bravo Urbina Paula, "Estudio psicológico y de las variables de Clima Laboral, Calidad de Trato, Satisfacción Subjetiva, Niveles de Estrés y Depresión, en el personal de Enfermería de la Unidad de Tratamiento Intensivo Quirúrgico del Hospital Clínico de la Pontificia Universidad Católica de Chile" Santiago de Chile. 2002.

Calcina Caceres, M. H. (2012). "Clima Organizacional y Satisfacción Laboral en enfermeros de unidades criticas del Hospital Nacional Dos de Mayo 2012".

Castillo Davila, N. F. (2014). Clima, Motivación Intrínseca y Satisfacción Laboral en Trabajadores.

Chiang Vega, M. M., \& Ojeda Hidalgo, J. (s.f.). Estudio de la relación entre Satisfacción Laboral y el Desempeño.

Chiavenato, Idalberto; (2007) "Administración de Recursos Humanos"; Octava Edición; Mc Graw Hill; México.

Fernández L., Paravic K.,"Nivel de Satisfacción Laboral en Enfermeras de Hospitales Públicos y Privados de la Provincia de Concepción, Chile" en Chile, 2003.

Jiménez Coronado, Ludmin (2010). Manual de regímenes Laborales. (1ra. Edición). Perú. Instituto Pacifico SAC: 2010.

Neves Mujica, Javier (2004). Introducción al Derecho Laboral. (1ra edición). Perú. Fondo editorial PUCP.

Pablos Gonzales, M. d. (2016). Estudio de Satisfacción Laboral y Estrategias de Cambio de las Enfermeras en los Hospitales Públicos de Badajoz y Cáceres.

Palma S.(1999). Elaboración y estandarización de la escala de satisfacción laboral (SL-SPC) en una muestra de trabajadores de Lima Metropolitana. Revista de la Facultad de Psicología U.R.P. (vol. IX n. ${ }^{\circ}$ 1).

Prado Alvarez, C. G. (2015). Relación entre clima laboral y desempeño laboral en los trabajadores administrativos de la Universidad Cesar Vallejo de Trujillo.

Quispe Aquino, N. (2015). Clima Organizacional y Satisfacción Laboral en la Asociación para el desarrollo empresarial en Apurímac, Andahuaylas, 2015. 